\title{
Probing the Top Quark Flavour-Changing Neutral Current at a Future Electron-Positron Collider
}

\author{
Hoda Hesari, ${ }^{1}$ Hamzeh Khanpour, ${ }^{1,2}$ \\ Morteza Khatiri Yanehsari, ${ }^{1,3}$ and Mojtaba Mohammadi Najafabadi ${ }^{1}$ \\ ${ }^{1}$ School of Particles and Accelerators, Institute for Research in Fundamental Sciences (IPM), P.O. Box 19395-5531, Tehran, Iran \\ ${ }^{2}$ Department of Physics, Mazandaran University of Science and Technology, P.O. Box 48518-78413, Behshahr, Iran \\ ${ }^{3}$ Department of Physics, Ferdowsi University of Mashhad, P.O. Box 1436, Mashhad, Iran
}

Correspondence should be addressed to Mojtaba Mohammadi Najafabadi; mojtaba@ipm.ir

Received 25 June 2014; Revised 2 September 2014; Accepted 10 September 2014; Published 3 November 2014

Academic Editor: Stephen Pate

Copyright (C) 2014 Hoda Hesari et al. This is an open access article distributed under the Creative Commons Attribution License, which permits unrestricted use, distribution, and reproduction in any medium, provided the original work is properly cited. The publication of this article was funded by SCOAP ${ }^{3}$.

We present a study to examine the sensitivity of a future $e^{-} e^{+}$collider to the anomalous top flavour-changing neutral current (FCNC) to the gluon. To separate signal from background a multivariate analysis is performed on top quark pair and background events, where one top quark is considered to follow the dominant standard model (SM) decay, $t \rightarrow W b$, and the other top decays through FCNC, $t \rightarrow q g$, where $q$ is a $u$ - or a $c$-quark. The analysis of fully hadronic FCNC decay of the $t \bar{t}$ pair is also presented. The $95 \%$ confidence level limits on the top quark anomalous couplings are obtained for different values of the center-of-mass energies and integrated luminosities.

\section{Introduction}

The top quark, which is the heaviest known elementary particle up to now, plays a special role in search for new physics beyond the standard model (SM) in particular through precise measurement of its couplings with other particles. The large mass of the top quark, $M_{\text {top }}=173.34 \pm 0.27$ (stat) \pm 0.71 (syst) [1], that is close to the scale of electroweak symmetry breaking and its interactions with other particles such as the Higgs boson make it an excellent object to investigate the validity of the SM. The anomalous interactions of the top quark can occur in various flavour-changing neutral current (FCNC) processes like $t \rightarrow q X$, where $X=q, \gamma, Z$, or Higgs. In [2], the anomalous tq $\gamma$ and $t q Z$ have been probed at a future electron-positron collider. In the present study, we focus on the top quark FCNC interactions involving the top quark, a light quark $q$, ( $u$ - or $c$-quark), and a gluon. In the SM, the FCNC transition of $t \rightarrow q g(q=u, c)$ is forbidden at tree level due to the Glashow-IliopoulosMaiani (GIM) mechanism [3] and only can proceed through the loop corrections. In the SM framework, the loop-level branching ratio for $t \rightarrow q g(q=u, c)$ is of the order of $10^{-12}[4,5]$. Clearly, a lot of data is needed to enable us to observe such a decay process and measure this small branching ratio. Various models beyond the SM could lead to a very large increase in FCNC processes involving the top quark. Thus, any evidence of such processes will indicate the existence of new physics. In models beyond SM such as MSSM, Technicolor, extra dimensions models higher branching ratios up to $10^{-3}-10^{-5}$ are predicted [6-10] which can be tested by present high energy experiments. There are several phenomenological studies in search for the anomalous tqg couplings at the Tevatron and LHC and other experiments through different channels [11-14]. At present the best and upto-date experimental limits on the tqg branching fractions come from the direct top production process at the Large Hadron Collider (LHC) by the ATLAS Collaboration, $\mathrm{Br}(t \rightarrow$ $u g)<3.1 \times 10^{-5}$ and $\operatorname{Br}(t \rightarrow c g)<1.6 \times 10^{-4}$ at a centerof-mass energy of $\sqrt{s}=8 \mathrm{TeV}$ corresponding to an integrated luminosity of $\mathscr{L}_{\text {int }}=14.2 \mathrm{fb}^{-1}[15,16]$.

It is expected that the future $\mathrm{TeV}$ scale linear colliders such as Compact Linear Collider (CLIC) or International 
Linear Collider (ILC) would complete the LHC probes and even in some processes can improve the measurements and limits. The high luminosity and clean experimental environments of the $\mathrm{TeV}$ scale $e^{-} e^{+}$collider make it an excellent precision machine for the investigation of the top quark properties. It also provides us with an important opportunity for precise measurements of the FCNC couplings in top quark sector [2, 17]. For example, in $[2,17]$ it has been shown that the branching ratios of the top quark decay into a photon and a $Z$ boson can be measured up to the order of $10^{-6}$ at a linear electron-positron collider.

The $e^{-} e^{+}$collider, CLIC, is designed to operate with the center-of-mass energies of $\sqrt{s}=0.5,1.5$, and $3 \mathrm{TeV}$ corresponding to total luminosity of $L=2.3,3.2$, and $5.9 \times$ $10^{-34} \mathrm{~cm}^{-2} \mathrm{~s}^{-1}$, respectively [18-21]. The design of ILC is to work at the center-of-mass energies from $\sqrt{s}=0.25 \mathrm{TeV}$ to $0.5 \mathrm{TeV}$ with the option of upgrading to $1 \mathrm{TeV}$. The plan for the ILC instantaneous luminosities is to reach $10^{-33}$. $10^{-34} \mathrm{~cm}^{-2} \mathrm{~s}^{-1}[22,23]$. One of the main differences between the ILC and CLIC is the difference in the luminosity spectrum (LS) of these machines. The ILC luminosity spectrum has a narrower peak of luminosity. This leads to an increase of the total luminosity and consequently reducing the statistical uncertainty in the measurements.

In this work, we study the sensitivity of a future electronpositron collider (CLIC or ILC) to the anomalous top flavourchanging neutral current (FCNC) to the gluon, $t-q-g$. To separate signal from backgrounds, a multivariate technique is used. We consider $e^{-} e^{+} \rightarrow t \bar{t} \rightarrow q g \ell^{+} \nu_{\ell} b\left(\bar{q} g \ell^{-} \bar{v}_{\ell} \bar{b}\right)$ (semileptonic) and $e^{-} e^{+} \rightarrow t \bar{t} \rightarrow q \bar{q} g g$ (full-hadronic) separately to search for the anomalous FCNC interactions in $t-q-g$ vertex. The analysis can also be done in the fullhadronic case with one of the top quarks decays into $t \rightarrow$ $W b \rightarrow j j j$ and the other top decays through anomalous couplings. Because of the large background contribution the results would be better than the semileptonic case; therefore we do not perform the analysis for this decay mode. We consider the center-of-mass energies of $0.5,1$, and $1.5 \mathrm{TeV}$, and for these energies we analyse two cases, semileptonic and fully hadronic decays of top quark.

The presented paper is organised as follows. In Section 2 , we introduce the theoretical formalism which describes the FCNC processes. Section 3 provides a full detailed description of the semileptonic channel in search for the tqg FCNC. The event selection and the methods of event classification into signal- and background-like events using a multivariate analysis are also discussed in this section. Our fully hadronic analysis is presented in Section 4. The results of the investigated FCNC processes, including expected sensitivities on the anomalous couplings and corresponding branching fractions, are given in Section 5. Discussions on some detector effects and systematic uncertainties are also presented in Section 5. Section 6 contains a summary and conclusions of the analysis.

\section{Theoretical Formalism}

In this section, we give a brief overview of the theoretical framework for top FCNC which this analysis is based on. In this work, to describe the FCNC couplings amongst the top quark, a light quark, and a gluon (tqg) an effective Lagrangian approach is used. The FCNC anomalous interaction in the vertex of $t$ g can be written as follows [11, 24-27]:

$$
\mathscr{L}_{\text {eff }}=\sum_{q=u, c} \frac{1}{\Lambda} g_{s} \kappa_{t q g} \bar{t} \sigma^{\mu \nu} T^{a} \chi q G_{\mu \nu}^{a}+h . c .,
$$

where the $\kappa_{\text {tqg }}$ with $q=u, c$ are dimensionless real parameters that present the strength of the anomalous couplings and strong coupling constant is denoted by $g_{s}$. In (1), $T^{a}=\lambda^{a} / 2$ where $\lambda^{a}$ are the Gell-Mann matrices, $\Lambda$ is the new physics scale, $G_{\mu \nu}^{a}$ is the gluon field tensor, and $\sigma^{\mu \nu}=(i / 2)\left[\gamma^{\mu}, \gamma^{\nu}\right]$. In the effective Lagrangian $\chi=f_{q}^{L} P_{L}+f_{q}^{R} P_{R}$ with $P_{L}\left(P_{R}\right)$ operators perform the left- (right-) handed projection and $f_{q}^{L(R)}$ are chiral parameters normalized to $\left|f_{q}^{L}\right|^{2}+\left|f_{q}^{R}\right|^{2}=1$. In Figure 1, we show the top pair production cross section times branching ratios of one top decays anomalously into $q+g$ and another one decays leptonically (electron and muon) as well as the top pair production cross section times the branching ratios of both tops decay anomalously into $q+g$. It is presented for different center-of-mass energies, $\sqrt{s}=0.5,1$, and $1.5 \mathrm{TeV}$, versus the anomalous coupling $\kappa_{t q g} / \Lambda$. As it can be seen the $\sigma\left(e^{-} e^{+} \rightarrow t \bar{t} \rightarrow q g \ell^{-} \nu \bar{b}\left(\bar{q} g \ell^{+} \nu b\right)\right)\left(\kappa_{t q g} / \Lambda=0.02 \mathrm{TeV}^{-1}\right)=$ $22.2 \mathrm{fb}$ and the $\sigma\left(e^{-} e^{+} \rightarrow t \bar{t} \rightarrow q g \bar{q} g(\bar{q} g q g)\right)\left(\kappa_{t q g} / \Lambda=\right.$ $\left.0.02 \mathrm{TeV}^{-1}\right)=9.6 \mathrm{fb}$ for the center-of-mass energy of $0.5 \mathrm{TeV}$. In order to calculate the cross section and simulate the events for the analysis, the FCNC effective Lagrangian has been implemented in the FeynRules package [28, 29]; then the model has been imported to a Universal FeynRules Output (UFO) module [30] and finally inserted to the MadGraph 5 [31]. The values of the cross sections are found to be in agreement with CompHEP package $[32,33]$.

\section{Semileptonic Channel}

This section presents the analysis of our signal and the corresponding backgrounds of semileptonic channel of $t \bar{t}$ events at the $e^{-} e^{+}$collider. In this channel, one of the top quarks decays through SM decay mode of $t \rightarrow W b \rightarrow$ $\ell \nu_{\ell} b(\ell=e, \mu)$ and the other one is considered to decay through FCNC into $q+g$, where $q$ is $u$ - or $c$-quark. The hadronic final states of $W$ boson have larger background contribution which would not lead to better sensitivity with respect to the semileptonic channel. Therefore, the leptonic decay modes of the $W$ boson that provide cleaner signature are considered. The final state signal topology consists of an energetic lepton, neutrino (appears as missing momentum), and three hadronic jets. One of the jets is originated from a $b$-quark. The representative Feynman diagram for the signal process is depicted in Figure 2.

Based on the expected signature of the signal events, the background topology is therefore given by $W^{ \pm} j j j \rightarrow$ $\ell^{ \pm} \bar{\nu}_{\ell} j j j$. In order to investigate the possibility of separating signal from the background events, we use Monte Carlo (MC) simulation. The MC generation of the signal sample, $t \bar{t} \rightarrow$ $b \ell v_{\ell} q g$, is done with CompHEP $[32,33]$ and the complete set of $\ell \nu+3 j$ backgrounds including the SM process $t \bar{t} \rightarrow \ell \bar{\nu}_{\ell} j j j$ 


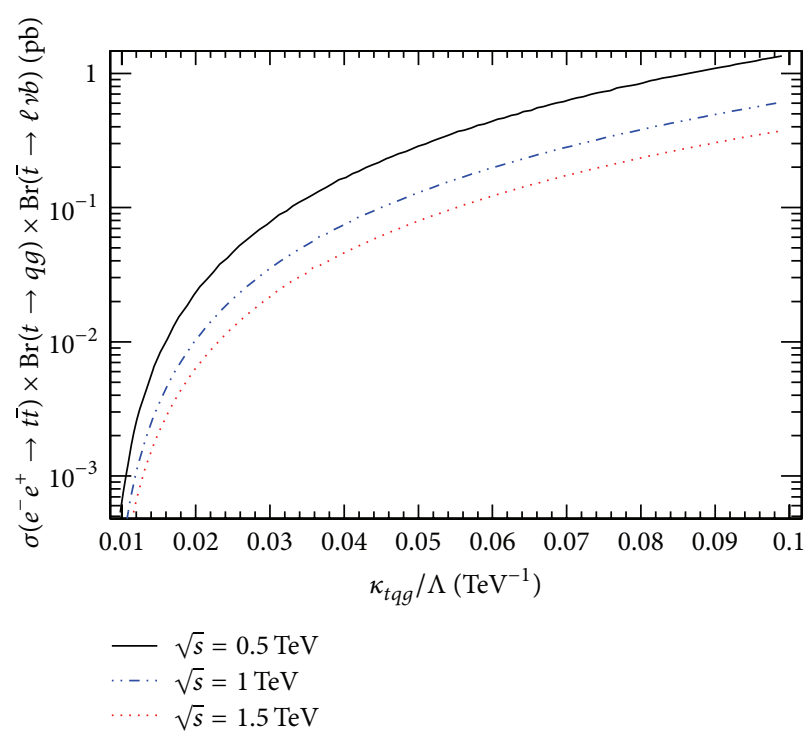

(a)

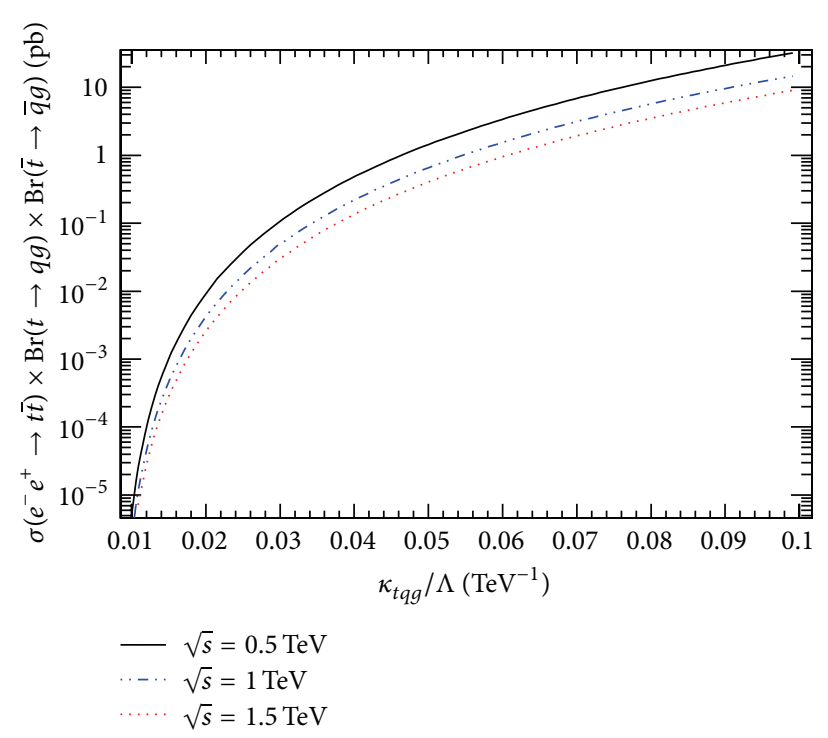

(b)

FIGURE 1: The cross section times branching ratio of one (a) and both (b) of the top quarks decay anomalously into $q+g$ as a function of the anomalous coupling $\kappa_{\text {tug }} / \Lambda$ for $\sqrt{s}=0.5,1$, and $1.5 \mathrm{TeV}$.

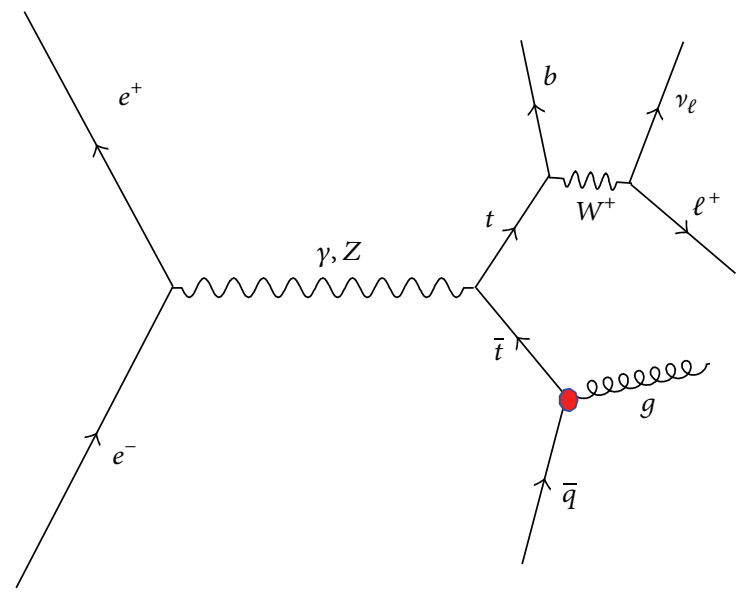

Figure 2: The representative Feynman diagram for the signal process in the semileptonic channel.

is generated using MadGraph [31]. The symbol $j$ represents any jet that originates from quarks and gluon. To account for the resolution of detectors, we apply energy smearing effects to the final state particles according to the following relations $[19,34]$ :

$$
\frac{\Delta E_{\text {jet }}}{E_{\text {jet }}}=\frac{40 \%}{\sqrt{E_{\text {jet }}}} \oplus 2.5 \%, \quad \frac{\Delta E_{\ell}}{E_{\ell}}=\frac{15 \%}{\sqrt{E_{\ell}}} \oplus 1 \%,
$$

where $E_{\text {jet }}$ and $E_{\ell}$ represent the energy of the jets and leptons, respectively. The energies are in $\mathrm{GeV}$ and the terms are added in quadrature. The jet energies are smeared according to a Gaussian distribution. We smear the energies of muons similar to the electrons for simplicity. Notice that better resolutions for leptons and jet lead to improvement of the results.
We apply the detector acceptance cuts on the transverse momenta of leptons (jets), $p_{T}>20(30) \mathrm{GeV}$, and pseudorapidities, $|\eta|<2.5$. In order to have well-isolated objects, it is required that the distances in $(\eta, \phi)$ space between each of the two objects satisfy $\Delta R_{i j}=\sqrt{\left(\eta_{i}-\eta_{j}\right)^{2}+\left(\phi_{i}-\phi_{j}\right)^{2}}>$ 0.4 . It is assumed that the presence of a high- $p_{T}$ electron or muon plays would be sufficient for triggering the signal events. Now, the signal events are reconstructed as follows. A full reconstruction of the $W$ boson four-momentum $\left(p_{W}\right)$ is needed to be able to reconstruct the semileptonic decaying top that is the combination of the reconstructed $W$ and $b$-jet, $M_{W b \text {-jet }}^{\text {rec }}$. It should yield a distribution consistent with the top quark invariant mass, $M_{\text {top }}$.

Due to undetected neutrino which leaves no track in the detector, we have difficulties in reconstruction of the $W$ boson. The transverse components of the neutrino momentum $\left(p_{T}^{v}\right)$ can be identified by the missing transverse momentum of the events. The longitudinal component of the momentum of the neutrino, $p_{z}^{v}$, can be found by solving the following quadratic equation:

$$
p_{W}^{2}=\left(p_{\ell}+p_{\nu}\right)^{2}=M_{W}^{2},
$$

where we put a mass constraint on $W, M_{W}=80.4 \mathrm{GeV}$. Solving the above quadratic equation allows us to obtain the longitudinal component of the neutrino momentum. This equation has up to two real solutions. In the case of having two real solutions, the one with minimum absolute value is taken. For the events with complex solution only the real part of the solution is considered as the $z$-component of the neutrino momentum.

We assume a $b$-tagging efficiency of about $60 \%$ for $b$-jets, $5 \%$ for $c$-quarks, and $1 \%$ for lighter quarks to be mistagged as $b$-quark jets $[35,36]$. In order to reconstruct the invariant 


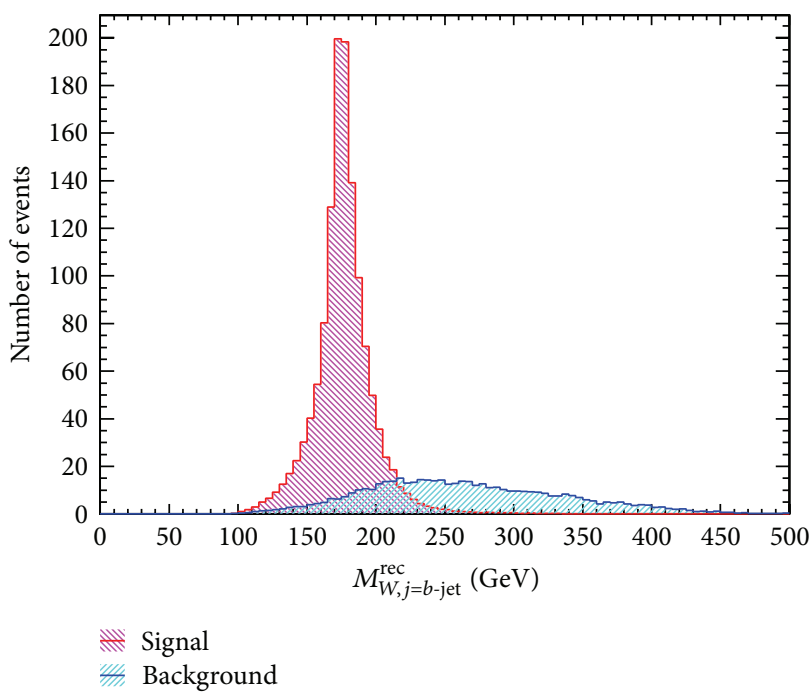

(a)

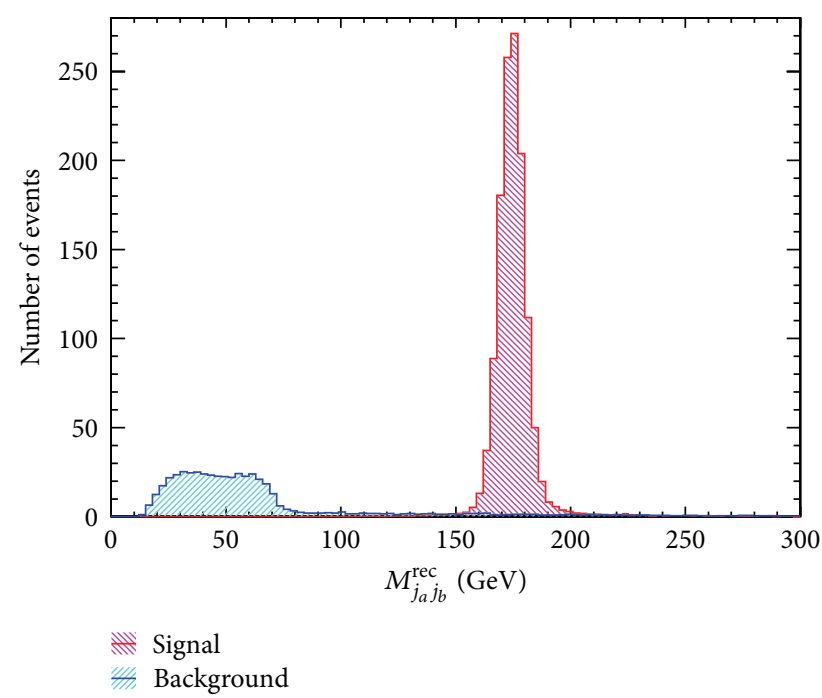

(b)

Figure 3: Reconstructed top quark mass distributions of the $W$ boson and $b$-jet $M_{W, j_{c}=b \text {-jet }}^{\text {rec }}$ (a) and the other two-jet $M_{j_{a} j_{b}}^{\text {rec }}$ (b) after the preselection cuts, at $\sqrt{s}=0.5 \mathrm{TeV}$. The number of events is normalized to $100 \mathrm{fb}^{-1}$ integrated luminosity of data and for the signal $\kappa_{\text {tqg }} / \Lambda=$ $0.02 \mathrm{TeV}^{-1}$.

mass of the semileptonic decaying top quark, $M_{\text {top }}$, we require a completely reconstructed $W$ boson and $b$-tagged jet. The anomalously decaying top quark will be reconstructed by combination of the two other remaining jets, which are not tagged as $b$-jets. The reconstructed invariant masses of both top quarks should have mass closest to the physical top quark mass, $M_{\text {top }}$. In some events, there can be more than one $b$ tagged jet. To make the correct combination of the jets, the event reconstruction is completed by minimizing $\chi_{a b c}^{2}$ defined as

$$
\chi_{a b c}^{2}=\left(M_{j_{a} j_{b}}^{\mathrm{rec}}-M_{\mathrm{top}}\right)^{2}+\left(M_{j_{c} W}^{\mathrm{rec}}-M_{\mathrm{top}}\right)^{2}+\Delta R_{j_{c} \ell}^{2}
$$

where $M_{j_{a} j_{b}}^{\text {rec }}$ is the reconstructed mass of the anomalously decaying top quark and $M_{j_{c} W}^{\mathrm{rec}}$ is the reconstructed mass of the top quark decaying through SM. $\Delta R_{j_{c} \ell}^{2}$ is the angular distance between lepton and jets. It is expected that the jet originating from the semileptonic top quark decays to be too close to the charged lepton. Various combinations of $\chi_{a b c}$ are made and the one with the minimum $\chi^{2}$ is chosen. The minimum value of $\chi^{2}$ implies that the reconstructed particles fit the requirement of coming from FCNC or SM top quark decay. The reconstructed top quark mass distributions, $M_{j_{a} j_{b}}^{\text {rec }}$ and $M_{W, j_{c}=b \text {-jet}}^{\text {rec }}$, for signal and corresponding background at center-of-mass energy of $\sqrt{s}=0.5 \mathrm{TeV}$ are shown in Figure 3. In Table 1 (left side), we show the number of signal and background events before and after the kinematical cuts for an integrated luminosity of $100 \mathrm{fb}^{-1}$. In this table, the numbers are presented after including the $b$-tagging efficiency.

Certainly, a detailed background study is essential in order to separate the signal from the background events. We use TMVA $[37,38]$ as a toolkit for multivariate analysis
TABLE 1: The number of events before and after the kinematical cuts for signal and background at a center-of-mass energy of $0.5 \mathrm{TeV}$ and with $100 \mathrm{fb}^{-1}$ integrated luminosity of data for semileptonic and full hadronic top quarks decays (both tops decay anomalously). The $b$ tagging efficiencies have been included and for the signal we have set $\kappa_{\text {tqg }} / \Lambda=0.02 \mathrm{TeV}^{-1}$.

\begin{tabular}{lcccc}
\hline \multirow{2}{*}{ Decay mode } & \multicolumn{2}{c}{ Semileptonic } & \multicolumn{2}{c}{ Full-hadronic } \\
& Before cuts & After cuts & Before cuts & After cuts \\
\hline Signal & 2146.5 & 1297.4 & 480.0 & 354.0 \\
Background & 1743.2 & 500.0 & 58905.1 & 29211.3 \\
\hline
\end{tabular}

to separate signal from background. Indeed, a multivariate analysis technique is necessary because a single variable does not have sufficient discrimination power to separate signal from background events. Among the multivariate analysis techniques that are usually used to separate the signal events from the backgrounds, the boosted decision tree (BDT) method is chosen [39-41]. We choose the variables which have the most possible separation power between the signals from background events for the BDT input. The kinematical variables are selected as input to the BDT as follow: the reconstructed top quarks masses, the transverse momenta of the $b$-jet $p_{T}(b)$ and charged lepton $p_{T}(\ell)$ difference of the azimuthal angle between the reconstructed $W$ boson and the $b$-jet $\left|\Delta \phi_{W, b \text {-jet }}\right|$, the pseudorapidity distribution of $b$-jet $\left|\eta_{b \text {-jet }}\right|$, the invariant mass of the reconstructed $W$ boson $M_{\ell v}^{\text {rec }}$, and finally the angular separation between charged lepton and $b$ jet $\Delta R_{\ell, b \text {-jet }}$.

The variable list can be extended and more variables could be given in the BDT input for better discrimination between signal and backgrounds. The kinematic distributions of some used variables which have the most discrimination power are 


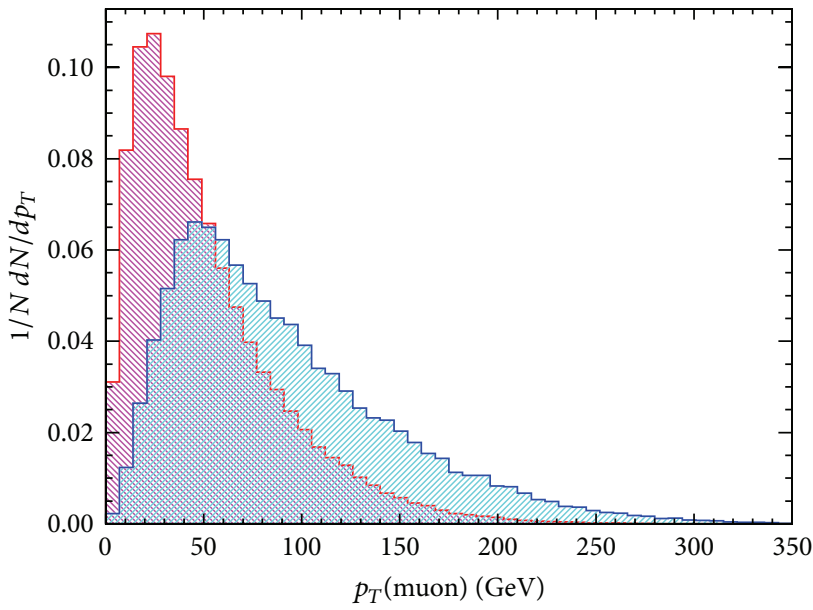

(a)

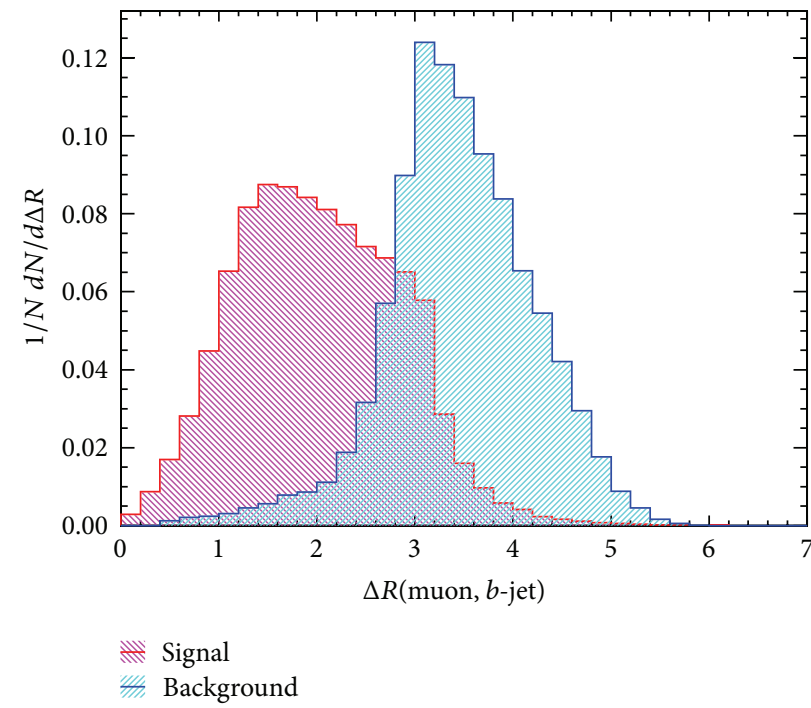

(c)

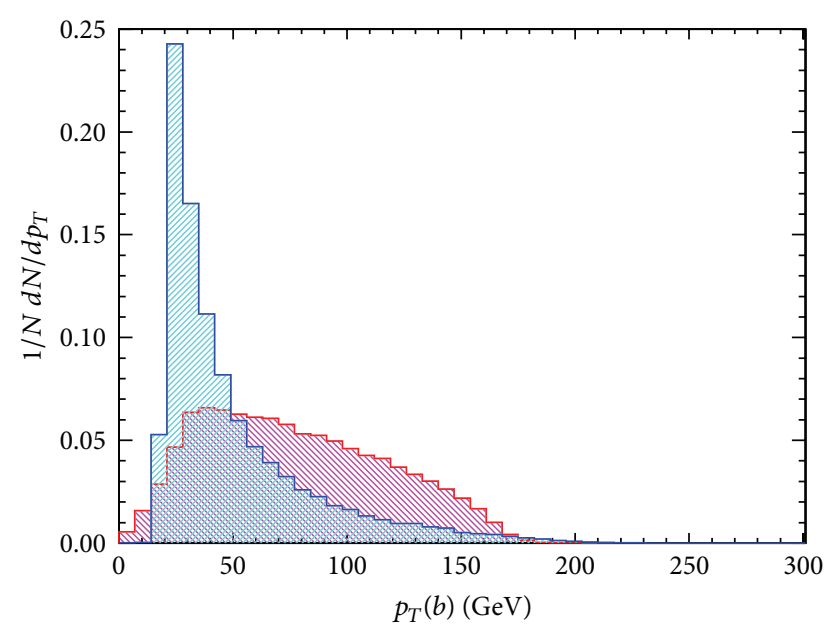

(b)

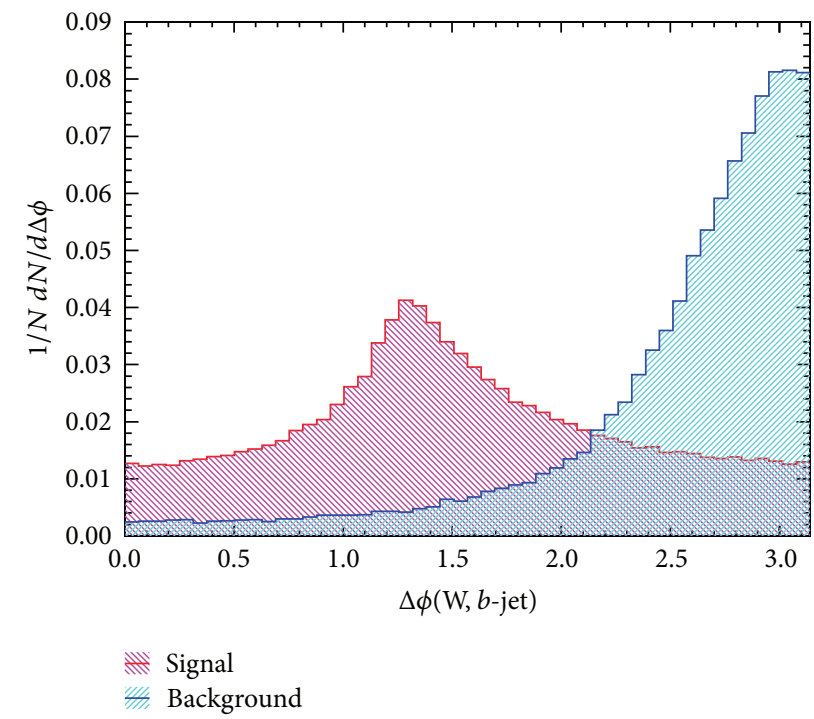

(d)

FIgURE 4: The kinematic distributions of four significance variables used as inputs to BDT in addition to the $W$ boson and top mass distributions. (a) The transverse momentum of the charged lepton $p_{T}(\ell)$ and (b) transverse momentum of the $b$-jet $p_{T}(b)$. (c) The angular separation between charged lepton and $b$-jet $\Delta R_{\ell, b \text {-jet }}$ and (d) the difference of the azimuthal angle between the reconstructed $W$ boson and the $b$-jet $\left|\Delta \phi_{W, b \text {-jet }}\right|$.

presented in Figure 4 before applying the acceptance cuts. These distributions are normalized to unity.

These variables are given to the BDT and the multivariate analysis is performed to achieve the best separation between signal and backgrounds and enhance signal significance. The test and training processes are done using a mixture of $50 \%$ of signal and $50 \%$ of background events. Due to the sensitivity of the BDT classifier to the statistical fluctuation of the training data sample, we use Adaptive Boosting algorithm to increase the performance. In order to avoid overtraining and to improve the quality of the analysis, the BDT builtin options such as Cost Complexity pruning methods are implemented during the training process. The goal is to find the best cuts that enhance the signal and reduce the background. Obtaining best cuts is generally done by finding the maximum value of the statistical significance, $n_{s} / \sqrt{n_{s}+n_{b}}$, where $n_{s}$ is the number of signal events and $n_{b}$ is the number of background events. By choosing the optimum cuts on the BDT output spectrum, we determine the number of selected signal and background events that provide the best signal significance. The results will be discussed in Section 5 .

\section{Fully Hadronic Decays of the $t \bar{t}$}

In pervious section, we discussed the signal and background where one of the top quarks decays through SM decay mode $t \rightarrow b W \rightarrow \ell v b$ and the other one is considered to decay through FCNC into $t \rightarrow q g$. In this section, we consider FCNC decay of both top quarks where the final state consists four jets at the center-of-mass energies of $\sqrt{s}=0.5,1$, and 1.5 TeV. The representative Feynman diagram for the signal process in the full-hadronic channel is depicted in Figure 5. 


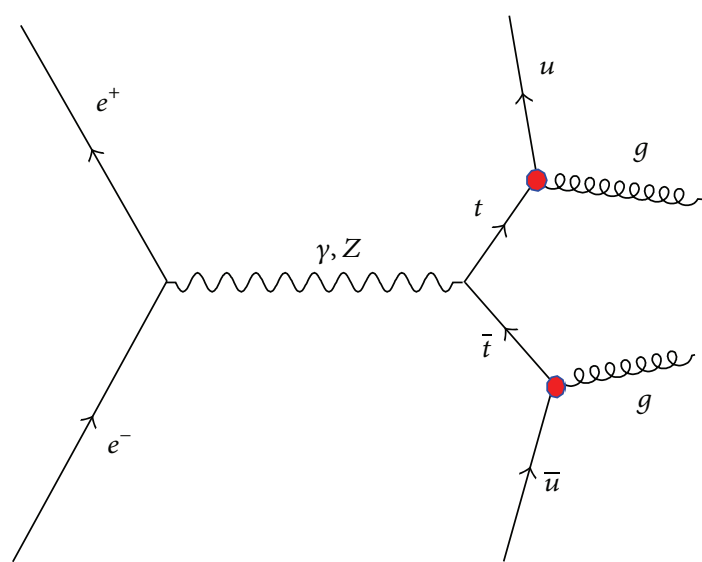

Figure 5: The representative Feynman diagram for the signal process in the hadronic channel.

It is worth mentioning that hadron colliders may not be a good area to study this fully hadronic process due to the extremely large QCD background contributions. The linear electron-positron colliders such as CLIC or ILC have a clean environment; consequently these fully hadronic final states can be probed at the CLIC or ILC easier than the hadron colliders.

The method of the channel is similar to semileptonic one that presented in the previous section. The MC generation of the signal sample is generated with CompHEP and the complete set of four-jet backgrounds is done using MadGraph 5.

Similar to the semileptonic case, to account for the resolution of the detectors a Gaussian energy smearing is performed on the final states jets. The jets are required to have transverse momenta greater than $30 \mathrm{GeV}$ within the pseudorapidity acceptance range of $|\eta|<2.5$. It is also required that $\Delta R_{i j}=\sqrt{\left(\eta_{i}-\eta_{j}\right)^{2}+\left(\phi_{i}-\phi_{j}\right)^{2}}>0.4$. The number of events before and after the kinematical cuts is shown in the right side of Table 1 for an integrated luminosity of $100 \mathrm{fb}^{-1}$. The reconstructed top quark mass distributions for signal and the corresponding background at center-ofmass energy of $\sqrt{s}=0.5 \mathrm{TeV}$ are shown in Figure 6 for an integrated luminosity of $100 \mathrm{fb}^{-1}$. The number of signal events in these figures has been multiplied by a factor of 10 .

Again we use the boosted decision tree (BDT) classifier of the TMVA package for discriminating signal from background events. For the BDT algorithm, the simulated events of the signal and background are split up into two similar samples for the training and test processes.

The input kinematical variables to the BDT are the reconstructed top quark masses $M_{\text {top }}^{\text {rec }}$, the transverse momentum of the highest $p_{T}$ jet, the corresponding pseudorapidity distribution $\left|\eta_{j}\right|$ of the highest $p_{T}$ jet, the angular separation $\Delta R_{j_{a} j_{b}}$ between the two jets, and the scalar transverse energy, $H_{T}$. The opening angles $\Delta \phi_{j_{a} j_{b}}$ between the directions of the final state jets are correlated with the mentioned variables so we neglected them. For the fully hadronic top quark reconstruction, we take the pair of jets which have an invariant mass closest to the nominal top quark mass as well as having smaller angular distance $\Delta R_{j_{a} j_{b}}$. In the analysis, an angular resolution of around $100 \mathrm{mrad}$ is assumed due to the expected high granularity design of the calorimeters of the future electron-positron collider [42].

In summary, in this section we concentrated on the channel of top pair production where both top quarks decay anomalously into two jets. After a rough detector simulation and applying the acceptance cuts, optimum kinematical variables are found and given to the BDT for discriminating between signal and backgrounds. In the next section, the limit on the branching fractions is given.

\section{Results}

With assuming of observation no signal events after performing the experiment or in other words if the number of observed events is equal to the number of expected background events, we proceed to set 95\% CL upper limit on the signal cross section. Limits on the cross section of signal are calculated with a CLs approach [43]. The RooStats [44] program is used for statistical data analysis for the numerical evaluation of the CLs limits. The program returns the 95\% CL upper limit on the signal cross section times branching ratios of the top quarks decays.

The sensitivity of the branching fractions as a function of the integrated luminosity for the future electron-positron collider at different center-of-mass energies is shown in Figures 7 and 8 for the semileptonic and fully hadronic analyses, respectively. As it can be seen from the figure, higher integrated luminosities lead to better bounds on the branching ratio up to around $500 \mathrm{fb}^{-1}$. The limits at the center-of-mass energy of $0.5 \mathrm{TeV}$ are better than the ones at 1 and $1.5 \mathrm{TeV}$ because of the larger cross sections at smaller energies. Comparing the semileptonic channel with the full-hadronic one, better sensitivity is achieved in the semileptonic channel. It is again due to the fact that in the full-hadronic channel statistics is poor with respect to the semileptonic one. The upper limits on the $\operatorname{Br}(t \rightarrow$ qg) at 95\% CL with $500 \mathrm{fb}^{-1}$ are 0.00117 and 0.0236 for the semileptonic and full-hadronic channels, respectively. It is interesting to mention here that the dependence of the expected upper limit on the integrated luminosity becomes weaker at luminosities larger than $500 \mathrm{fb}^{-1}$.

Now, the sensitivity of the results on the detector performance is discussed. In this analysis, almost all subdetectors are involved to identify and reconstruct leptons, jets, $b$ jets, and missing energy. Precise reconstruction of secondary vertex for an efficient $b$-tagging is necessary in this analysis to suppress the backgrounds and obtain a pure signal sample. The variation of $b$-tagging efficiency in this analysis by $10 \%$ leads to approximately $4 \%$ change in the expected upper limit on the branching ratio. The resolutions in measurement of the jet and lepton energies are less important than $b$-jet identification. Varying the resolution in jet and lepton energy measurement by $10 \%$ and $5 \%$ (2) leads to changing the upper limit on the branching fraction by less than $1 \%$.

In this analysis, we have calculated the cross section for the energy at the vertex of electron-positron. Therefore, further effects such as the initial state radiation (ISR) and the luminosity spectrum (LS) of the collider need to be 


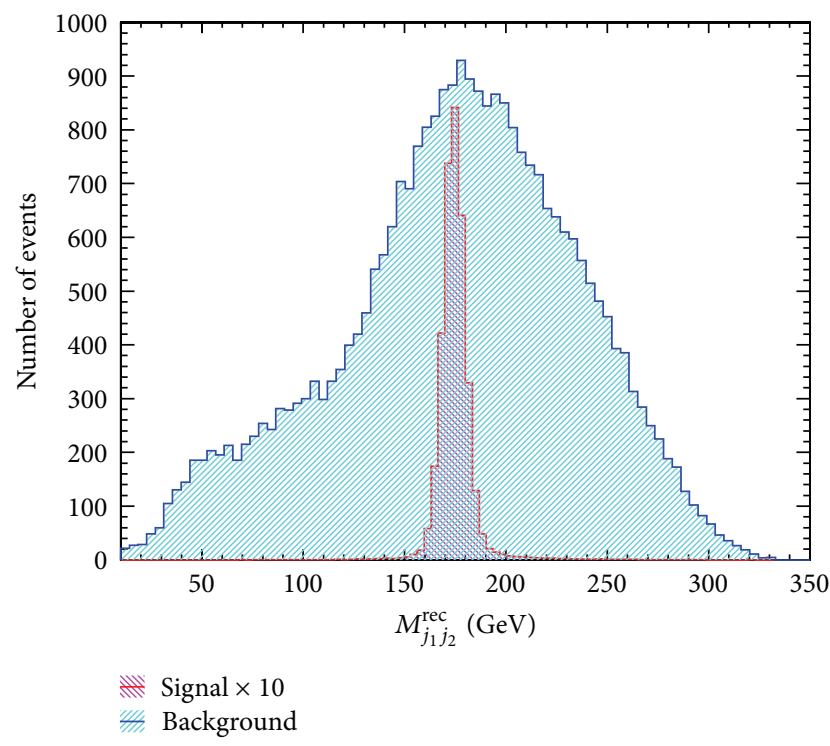

(a)

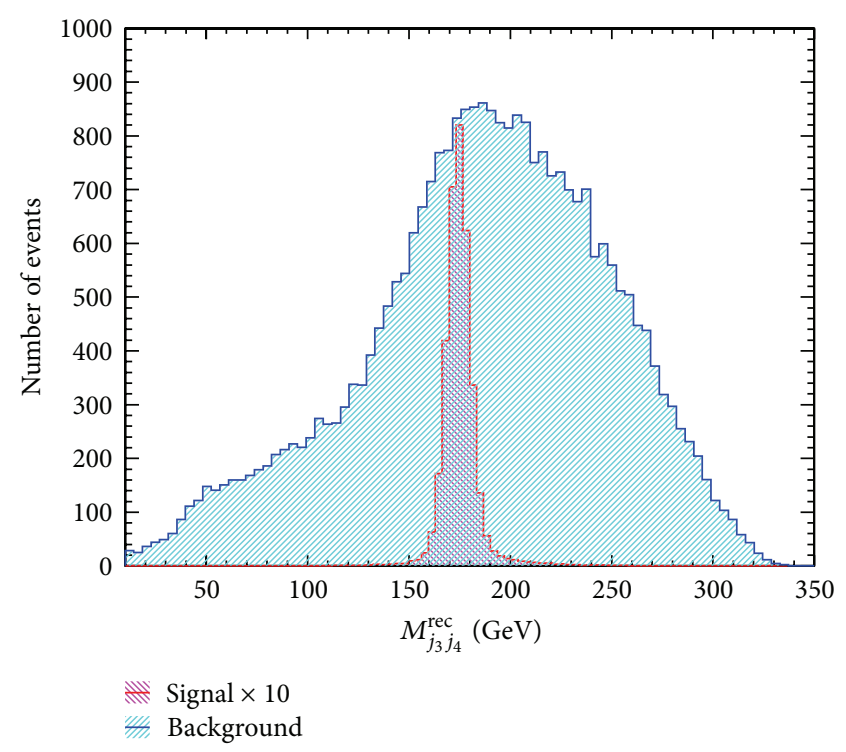

(b)

Figure 6: Reconstructed top quark mass distributions for the anomalous decay of both top quarks into a light quark and a gluon at $\sqrt{s}=$ $0.5 \mathrm{TeV}$. The distributions are normalized to $100 \mathrm{fb}^{-1}$ integrated luminosity of data and for the signal $\kappa_{\text {tqg }} / \Lambda=0.02 \mathrm{TeV}^{-1}$. The number of signal events is multiplied by a factor of 10 .

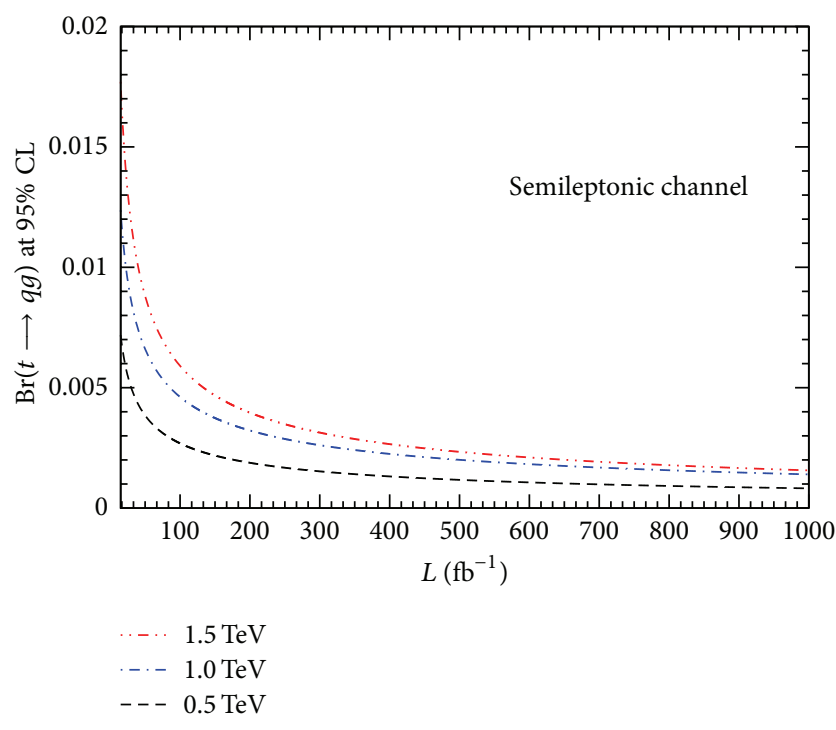

Figure 7: The 95\% CL upper limits for $\operatorname{Br}(t \rightarrow q g)$ as a function of integrated luminosity for $\sqrt{s}=0.5,1$, and $1.5 \mathrm{TeV}$ for the semileptonic analysis.

considered. Both the initial state radiation and the luminosity spectrum lead to reducing the cross section. We calculate the effect of ISR on the cross section at the center-of-mass energy of $500 \mathrm{GeV}$. The signal cross section decreases by around $2 \%$ which leads to losing the expected upper limit on the branching ratio from 0.00117 to 0.00118 with $500 \mathrm{fb}^{-1}$.

To have a more realistic analysis, the effects of the systematic uncertainties should be estimated. The uncertainties

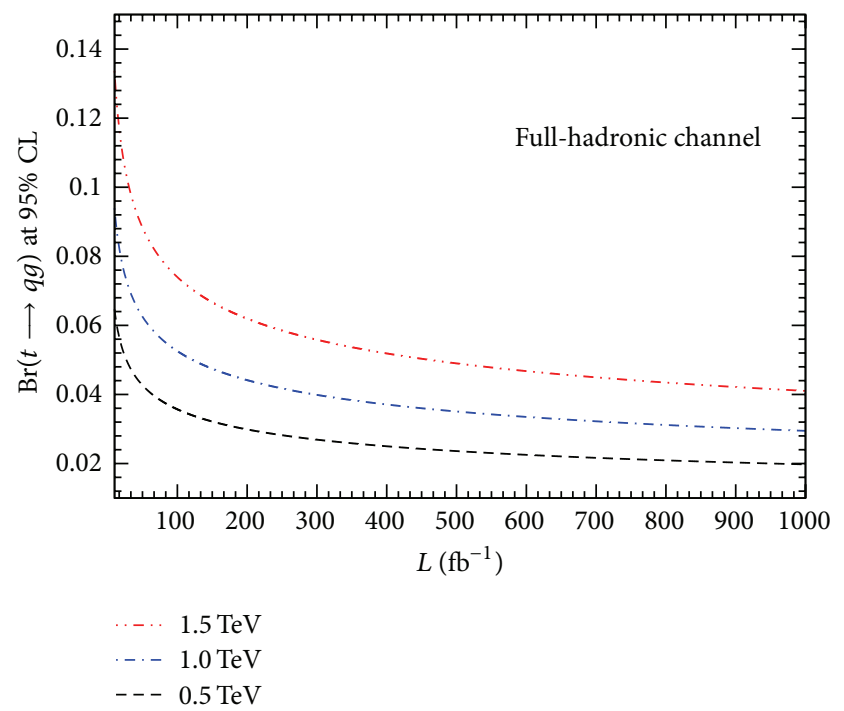

Figure 8: The 95\% CL upper limits for $\operatorname{Br}(t \rightarrow q g)$ as a function of integrated luminosity for $\sqrt{s}=0.5,1$, and $1.5 \mathrm{TeV}$ for the fully hadronic analysis.

can arise from jet energy scale, lepton energy, lepton reconstruction identification efficiencies, $b$-tagging efficiency, and uncertainties on the masses of the top quark and $W$ boson. We vary the $b$-tagging efficiency by $\pm 5 \%$. This leads to changing the expected upper limit by $1.5 \%$. To estimate the uncertainty from jet energy scale, we vary the energy of each jet by $2 \%$ and recalculate the limit. It results in a change of $0.5 \%$ on the expected upper limit. The uncertainties on the top quark and $W$ boson masses are calculated as follows. We 
TABLE 2: The 95\% CL upper limit on the $\operatorname{Br}(t \rightarrow q g)$ with the LHC $(8,14 \mathrm{TeV})$ and $e^{-} e^{+}(0.5 \mathrm{TeV})$ based on $100 \mathrm{fb}^{-1}$ integrated luminosity of data. The results of LHC8 are corresponding to $14.2 \mathrm{fb}^{-1}$.

\begin{tabular}{lcccccc}
\hline Collider & $\begin{array}{c}\text { LHC8 } \\
\left(14.2 \mathrm{fb}^{-1}\right)\end{array}$ & \multicolumn{3}{c}{ LHC14 $\left(100 \mathrm{fb}^{-1}\right)$} & $\begin{array}{c}e^{-} e^{+} \\
\left(100 \mathrm{fb}^{-1}\right)\end{array}$ \\
\hline Process & $2 \rightarrow 1$ & $2 \rightarrow 1$ & $2 \rightarrow 1$ & $t V$ & $t t, \overline{t t}$ & $t \bar{t}$ \\
Upper limit & $3.1 \times 10^{-5}$ & $10^{-6}$ & $10^{-5}$ & $10^{-5}$ & $10^{-3}$ & $10^{-3}$ \\
\hline
\end{tabular}

generate new signal samples with varied top mass $( \pm 1 \mathrm{GeV})$ and $W$ boson mass $( \pm 50 \mathrm{MeV})$ and redo the analysis. This leads to a change of $0.05 \%$ on the expected upper limit on the branching fraction of $t \rightarrow q g$.

5.1. Comparison with the LHC Results. So far, we have examined the future $e^{-} e^{+}$collider potential to probe the anomalous tqg in the decay of the top quark in top pair production. At hadron colliders, the anomalous tqg couplings can be probed either in top production or top decay. The best limits have been obtained in the production processes. There are different production channels to search for the anomalous tqg: (1) direct top quark production ( $\rightarrow 1$ process), (2) single top quark production $(2 \rightarrow 2)$, (3) double top pair $(t t, \overline{t t})$ production, and (4) top plus vector boson production $(t V)$ [45]. Currently, the strongest experimental limits on the tag branching fractions come from the direct top production $(2 \rightarrow 1$ process) at the LHC by the ATLAS Collaboration, $\operatorname{Br}(t \rightarrow u g)<3.1 \times 10^{-5}$ and $\operatorname{Br}(t \rightarrow c g)<1.6 \times 10^{-4}$ at a center-of-mass energy of $\sqrt{s}=8 \mathrm{TeV}$ corresponding to an integrated luminosity of $\mathscr{L}_{\text {int }}=14.2 \mathrm{fb}^{-1}$.

In [46], the anomalous tqg couplings have been probed in top decay at the Tevatron. The obtained upper limit on the branching ratio is $5(2.7) \times 10^{-3}$ with $10(30) \mathrm{fb}^{-1}$ of data. These limits are weaker in comparison with the limits that can be obtained from the production processes. The future LHC bounds at $14 \mathrm{TeV}$ center-of-energy using $100 \mathrm{fb}^{-1}$ of data using various processes are compared with the ones obtained in this work which are compared in Table 2. As it can be seen, among the all processes the $2 \rightarrow 1$ process provides the strongest limit $\left(10^{-6}\right)$. The limits that we have obtained in this study for $e^{-} e^{+}$collider are comparable with the ones that come out of the same-sign top $(t t, \overline{t t})$ production at the LHC and the ones from top decays in top pair events at the Tevatron.

\section{Summary and Conclusions}

In this paper, we have studied the signals of top quark flavorchanging neutral current in the vertex of $t q g$, where $q=u$ and $c$, at a future electron-positron collider. This study has been done by looking at the top pair production and at three different center-of-mass energies of $0.5,1$, and $1.5 \mathrm{TeV}$ in the top quarks decays. We have investigated two possible cases: first one is the case that one top quark decays anomalously to $q+g$ and another one follows SM decay to a $W$ boson and a $b$-quark and $W$ boson decays leptonically $\left(e^{-} e^{+} \rightarrow t \bar{t} \rightarrow\right.$ $\left.q g \ell^{+} v_{\ell} b\right)$. Second is that both top quarks decay anomalously through FCNC decay mode $\left(e^{-} e^{+} \rightarrow t \bar{t} \rightarrow q \bar{q} g g\right)$. Using the boosted decision tree (BDT) technique, we discriminate between signal and backgrounds. Then the CLs approach has been utilized to set upper limits on the branching ratio. The 95\% CL upper limit on the branching ratio using $500 \mathrm{fb}^{-1}$ of data at the center-of-mass energy of $0.5 \mathrm{TeV}$ is 0.00117 (0.0236) in semileptonic (full-hadronic) channel. It is shown that the limit is improved with the integrated luminosity up to around $500 \mathrm{fb}^{-1}$ and the dependence of the expected upper limit on the integrated luminosity becomes weaker at luminosities larger than $500 \mathrm{fb}^{-1}$.

We have found that sensitivity to the anomalous couplings decreases with increasing the center of energy of the collisions simply due to the decrease in the signal cross section with growing the center-of-mass energy. The expected bounds are comparable with the ones obtained from the double top production at the LHC $(t t, \overline{t t})$ and from the anomalous top decay in top pair events at Tevatron.

\section{Conflict of Interests}

The authors declare that there is no conflict of interests regarding the publication of this paper.

\section{Acknowledgments}

The authors are grateful to R. Goldouzian for valuable discussions. They acknowledge financial support of the School of Particles and Accelerators, Institute for Research in Fundamental Sciences (IPM).

\section{References}

[1] ATLAS Collaboration, CDF Collaboration, CMS Collaboration, and D0 Collaboration, "First combination of Tevatron and LHC measurements of the top-quark mass," http://arxiv.org/abs/1403.4427.

[2] J. A. Aguilar-Saavedra, "Top flavour-changing neutral coupling signals at a linear collider," Physics Letters B, vol. 502, no. 1-4, pp. 115-124, 2001.

[3] S. L. Glashow, J. Iliopoulos, and L. Maiani, "Weak interactions with lepton-hadron symmetry," Physical Review D, vol. 2, no. 7, pp. 1285-1292, 1970.

[4] G. Eilam, J. L. Hewett, and A. Soni, "Rare decays of the top quark in the standard and two-Higgs-doublet models," Physical Review D, vol. 44, article 1473, 1991.

[5] B. Mele, S. Petrarca, and A. Soddu, "A new evaluation of the $t \rightarrow$ $\mathrm{cH}$ decay width in the standard model," Physics Letters B, vol. 435, no. 3-4, pp. 401-406, 1998.

[6] S. Bejar, J. Guasch, D. Lopez-Val, and J. Sola, "FCNC-induced heavy-quark events at the LHC from supersymmetry," Physics Letters B, vol. 668, no. 5, pp. 364-372, 2008.

[7] J. Cao, Z. Heng, L. Wu, and J. M. Yang, " $R$-parity violating effects in top quark flavor-changing neutral-current production at LHC," Physical Review D, vol. 79, no. 5, Article ID 054003, 7 pages, 2009.

[8] R. Guedes, R. Santos, and M. Won, "Limits on strong flavor changing neutral current top couplings at the LHC," Physical Review D, vol. 88, Article ID 114011, 2013. 
[9] G. A. González-Sprinberg, R. Martínez, and J.-A. Rodriguez, "FCNC top quark decays in extra dimensions," The European Physical Journal C, vol. 51, no. 4, pp. 919-926, 2007.

[10] R. Coimbra, A. Onofre, R. Santos, and M. Won, "MEtop-a generator for single top production via FCNC interactions," The European Physical Journal C, vol. 72, article 2222, 26 pages, 2012.

[11] J. Gao, C. S. Li, L. L. Yang, and H. Zhang, "Searching for anomalous top quark production at the early LHC," Physical Review Letters, vol. 107, no. 9, Article ID 092002, 5 pages, 2011.

[12] J.-L. Agram, J. Andrea, E. Conte, B. Fuks, D. Gelé, and P. Lansonneur, "Probing top anomalous couplings at the LHC with trilepton signatures in the single top mode," Physics Letters $B$, vol. 725, no. 1-3, pp. 123-126, 2013.

[13] S. Khatibi and M. M. Najafabadi, "Probing the anomalous FCNC interactions in a top-Higgs boson final state and the charge ratio approach," Physical Review D, vol. 89, Article ID 054011, 2014.

[14] S. M. Etesami and M. M. Najafabadi, "Study of anomalous top quark flavor-changing neutral current interactions via the tW channel of single-top-quark production," Physical Review D, vol. 81, Article ID 117502, 2010.

[15] The ATLAS collaboration, ATLAS-CONF-2013-063, http://cds.cern.ch/record/1562777.

[16] E. Yazgan, "Flavor changing neutral currents in top quark production and decay," in Proceedings of the 6th International Workshop on Top Quark Physics (TOP '13), Durbach, Germany, September 2013.

[17] J. A. Aguilar-Saavedra and T. Riemann, "Probing top flavourchanging neutral couplings at TESLA," in Proceedings of the 5th Workshop of the 2nd ECFA/DESY Study on Physics and Detectors for a Linear Electron-Positron Collider, pp. 2428-2450, Obernai, France, October 1999.

[18] P. Lebrun, L. Linssen, A. Lucaci-Timoce et al., "The CLIC Programme: towards a staged e+e- linear collider exploring the terascale: CLIC conceptual design report," http://arxiv.org/abs/1209.2543.

[19] L. Linssen, A. Miyamoto, M. Stanitzki, and H. Weerts, "Physics and detectors at CLIC: CLIC conceptual design report," http://arxiv.org/abs/1202.5940.

[20] J. E. Brau, R. M. Godbole, F. R. L. Diberder et al., "The physics case for an e+e- linear collider," http://arxiv.org/abs/1210.0202.

[21] A. Aicheler, P. Burrows, M. Draper et al., A Multi-TeV Linear Collider Based on CLIC Technology : CLIC Conceptual Design Report, CERN, Geneva, Switzerland, 2012.

[22] H. Baer, T. Barklow, K. Fujii et al., "The international linear collider technical design report-volume 2: physics," http://arxiv.org/abs/1306.6352.

[23] T. Behnke, J. E. Brau, B. Foster et al., "The international linear collider technical design report-volume 1: executive summary," http://arxiv.org/abs/1306.6327.

[24] W. Buchmuller and D. Wyler, "Effective lagrangian analysis of new interactions and flavour conservation," Nuclear Physics B, vol. 268, pp. 621-653, 1986.

[25] E. Malkawi and T. M. P. Tait, “Top-quark-charm-quark strong flavor-changing neutral currents at the Fermilab Tevatron," Physical Review D, vol. 54, p. 5758, 1996.

[26] M. Hosch, K. Whisnant, and B. L. Young, "Direct top quark production at hadron colliders as a probe of new physics," Physical Review D, vol. 56, no. 9, pp. 5725-5830, 1997.

[27] J. A. Aguilar-Saavedra, "A minimal set of top anomalous couplings," Nuclear Physics B, vol. 812, no. 1, pp. 181-204, 2009.
[28] N. D. Christensen and C. Duhr, "FeynRules-Feynman rules made easy," Computer Physics Communications, vol. 180, pp. 1614-1641, 2009.

[29] C. Duhr and B. Fuks, "A superspace module for the FeynRules package," Computer Physics Communications, vol. 182, pp. 2404-2426, 2011.

[30] C. Degrande, C. Duhr, B. Fuks, D. Grellscheid, O. Mattelaer, and T. Reiter, "UFO-the universal FeynRules output," Computer Physics Communications, vol. 183, pp. 1201-1214, 2012.

[31] J. Alwall, M. Herquet, F. Maltoni, O. Mattelaer, and T. Stelzer, "MadGraph 5: going beyond," Journal of High Energy Physics, vol. 1106, p. 128, 2011.

[32] A. Sherstnev, A. Kryukov, E. Boos et al., "CompHEP status report (version 4.5)," in Proceedings of the 12th Conference on Advanced Computing and Analysis Techniques in Physics Research, PoS(ACAT08)008, Erice, Italy, November 2008.

[33] E. Boos, V. Bunichev, M. Dubinin et al., "CompHEP 4.4automatic computations from lagrangians to events," Nuclear Instruments and Methods in Physics Research A, vol. 534, pp. 250-259, 2004.

[34] J. Brau, Y. Okada, N. Walker et al., "ILC reference design report volume 1-executive summary," http://arxiv.org/abs/0712.1950.

[35] H. Baer, T. Barklow, K. Fujii et al., "The international linear collider technical design report-volume 2: physics," http://xxx.lanl.gov/abs/1306.6352.

[36] H. Abramowicz, A. Abusleme, K. Afanaciev et al., "Physics at the CLIC e+ e-Linear Collider-input to the Snowmass process 2013," http://arxiv.org/abs/1307.5288.

[37] J. Therhaag, PoS ICHEP, vol. 2010, p. 510, 2010.

[38] A. Hocker, P. Speckmayer, J. Stelzer et al., "TMVAToolkit for multivariate data analysis in ROOT," http://arxiv.org/abs/physics/0703039.

[39] B. P. Roe, H.-J. Yang, J. Zhu, Y. Liu, I. Stancu, and G. McGregor, "Boosted decision trees as an alternative to artificial neural networks for particle identification," Nuclear Instruments and Methods A, vol. 543, pp. 577-584, 2005.

[40] H.-J. Yang, B. P. Roe, and J. Zhu, "Studies of boosted decision trees for MiniBooNE particle identification," Nuclear Instruments and Methods in Physics Research A, vol. 555, no. 1-2, pp. 370-385, 2005.

[41] H.-J. Yang, B. P. Roe, and J. Zhu, "Studies of stability and robustness for artificial neural networks and boosted decision trees," Nuclear Instruments and Methods in Physics Research A, vol. 574, pp. 342-349, 2007.

[42] R. Pöschl and CALICE Collaboration, "R\&D for a SiW electromagnetic calorimeter for a future linear collider," Journal of Physics: Conference Series, vol. 293, no. 1, Article ID 012069, 2011.

[43] A. L. Read, "Modified frequentist analysis of search results (the CL(s) method)," in Proceedings of the Workshop on Confidence Limits, p. 81, Geneva, Switzerland, 2000.

[44] L. Moneta, K. Belasco, K. Cranmer et al., "The RooStats project," in Proceedings of the ACAT2010 Conference, 2010.

[45] M. Beneke, I. Efthymiopoulos, M. L. Mangano et al., "Top quark physics," in Proceedings of the CERN Workshop on Standard Model Physics (and More) at the LHC, pp. 419-529, Geneva, Switzerland, 1999.

[46] T. Han, K. Whisnant, B. L. Young, and X. Zhang, "Searching for $t \rightarrow c g$ at the Fermilab Tevatron," Physics Letters B, vol. 385, pp. 311-316, 1996. 

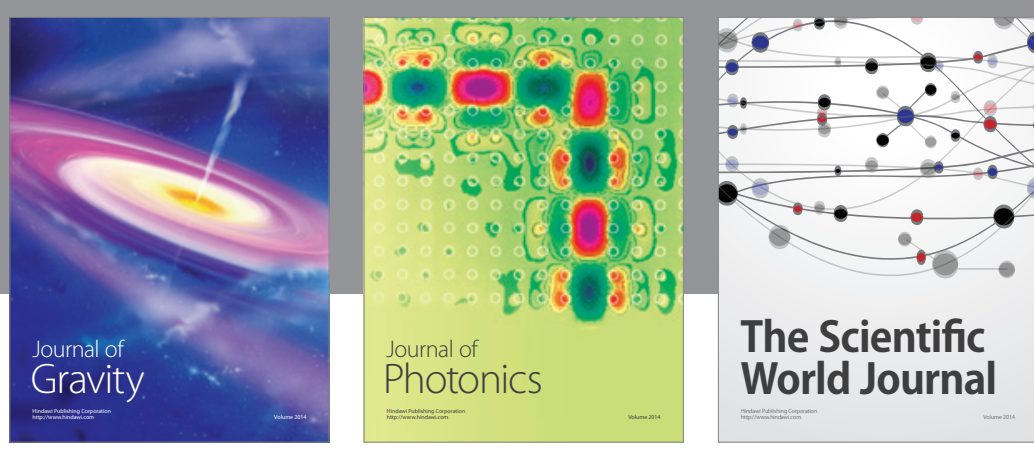

The Scientific World Journal
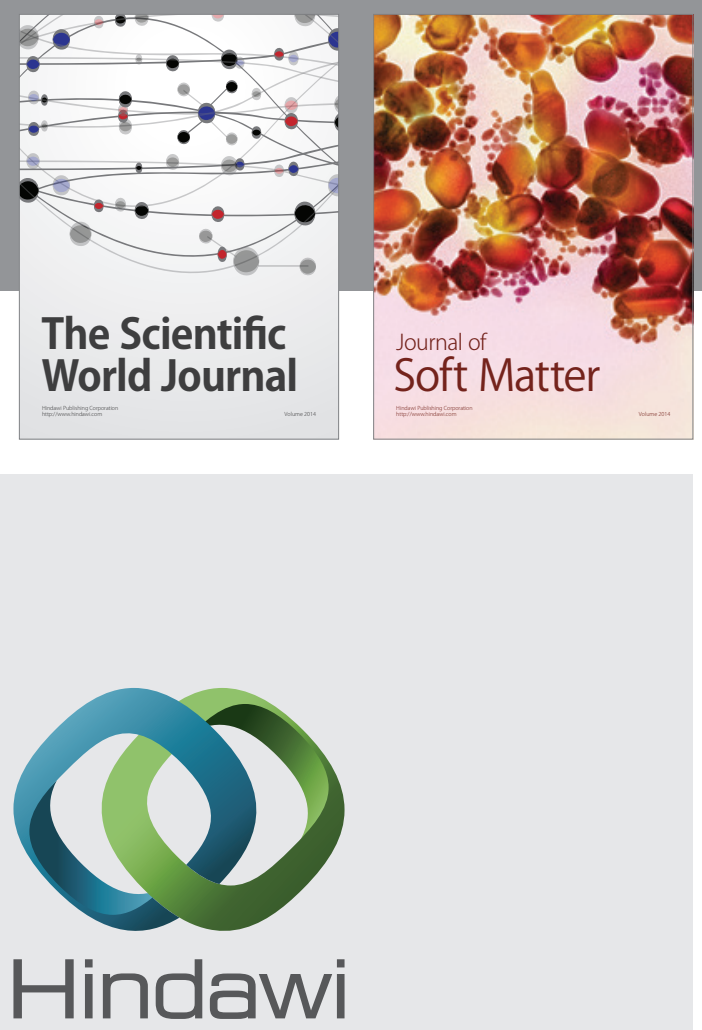

Submit your manuscripts at

http://www.hindawi.com

nternational Journal of

Statistical Mechanics
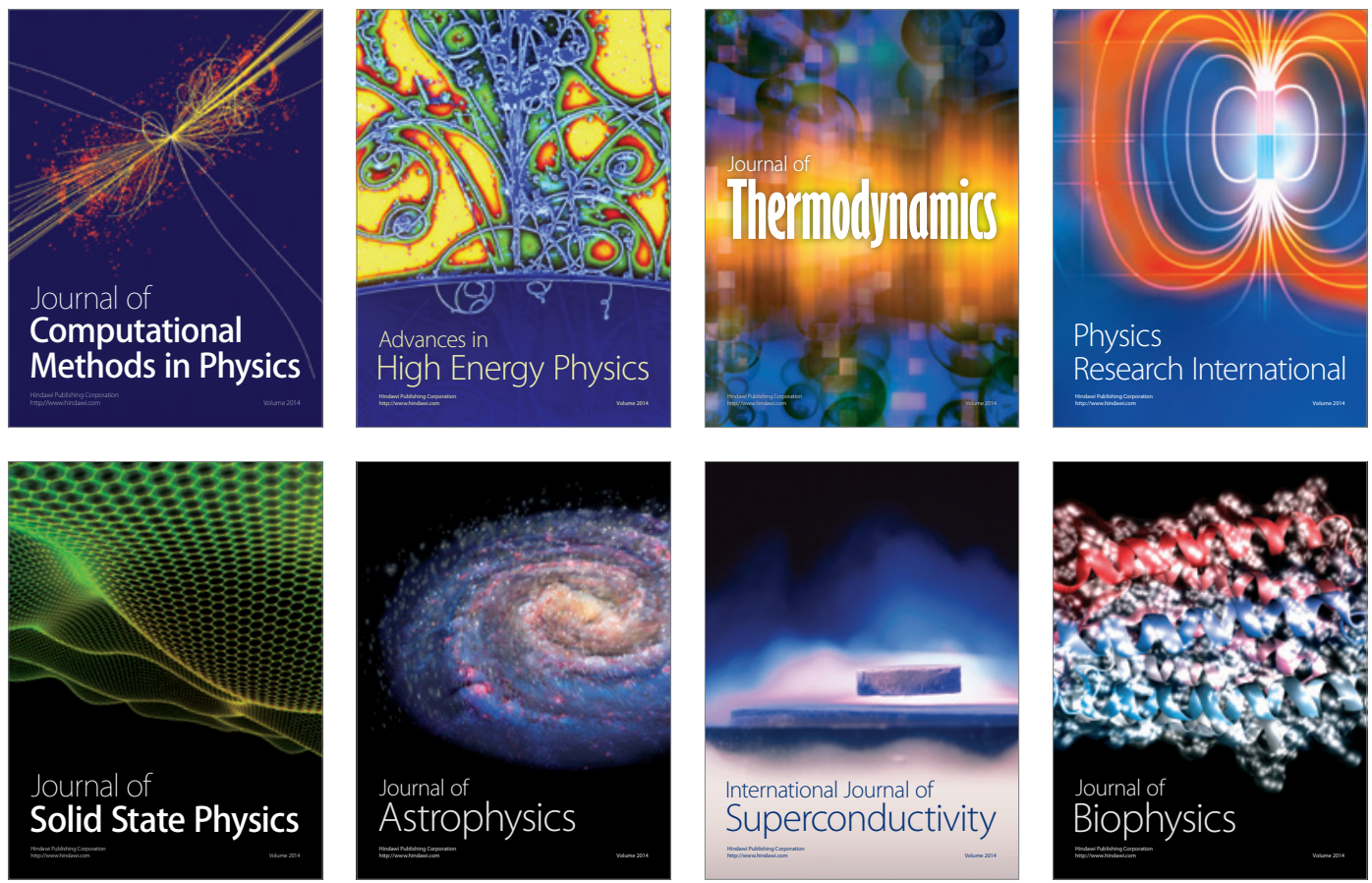
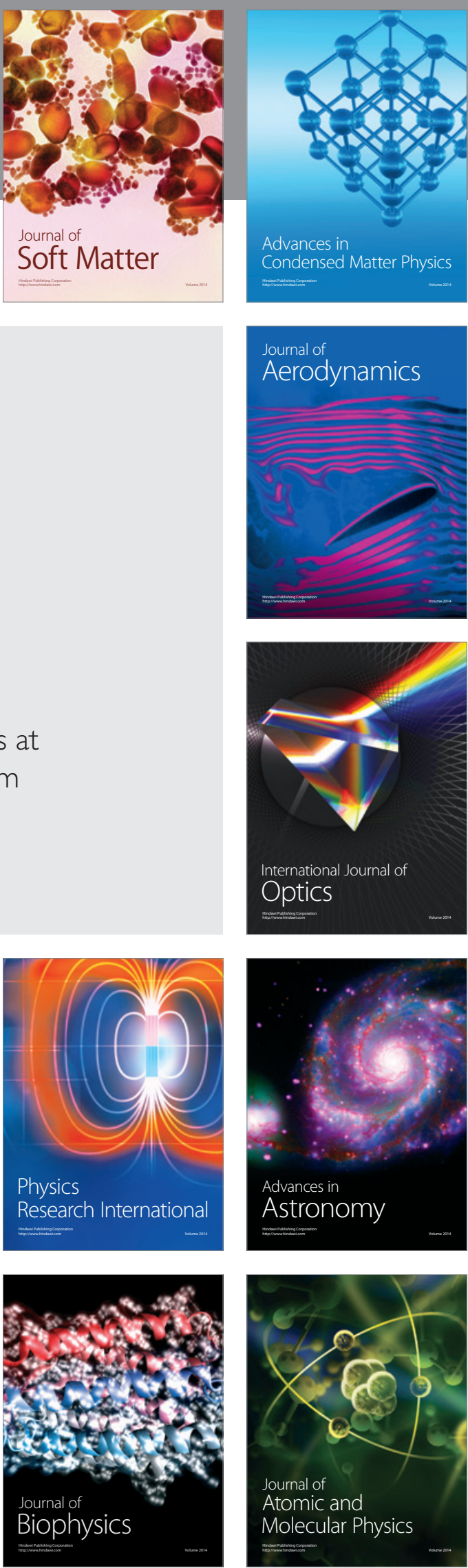